\title{
Non-Parametric Regression Method
}

National Cancer Institute

\section{Source}

National Cancer Institute. Non-Parametric Regression Method. NCI Thesaurus. Code C53239.

Non-parametric regression methods are aimed at describing a relationship between the dependent and independent variables without specifying the form of the relationship between them a priori. 